\title{
Efficient Prediction and Classification of Epileptic Seizures Using EEG Data Based on Univariate Linear Features
}

\author{
Syed Muhammad Usman*, Ali Hassan \\ College of Electrical and Mechanical Engineering, NUST Islamabad, Pakistan. \\ * Corresponding author. Tel.: 923320615294; email: muhammadusman81@ce.ceme.edu.pk \\ Manuscript submitted June 10, 2017; accepted August 10, 2017. \\ doi: $10.17706 /$ jcp.13.6.616-621
}

\begin{abstract}
Epilepsy is defined as seizures which happen due to disorder in brain functionality. It is certain kind of seizures which effect patient in more than twice in a day when patient loss its senses completely or partially for a short duration. Mostly people affected from epilepsy live in less developed or developing countries. Diagnosis of epilepsy at early stages is quite useful for better treatment of patients. Normal method of diagnosing epilepsy is to admit the patient into hospital and by viewing its EEG recordings. This method is not useful as this include viewing of EEG signals for many hours. In our paper, we propose an algorithm by using which we are able to predict epileptic seizure. There are three states of seizure that include pre-ictal state which is before the start of seizure, another is ictal state during which seizure is happening and there is also a post-ictal state which is after seizure. We have proposed an algorithm by using which we can predict seizure of affected patient i.e; pre-ictal state. We have applied our algorithm on publically available EEG dataset and it has been observed that average pre-ictal time is 34 minutes. It means that we are able to predict epilepsy 34 minutes on average before it actually starts. In this way, there is a sufficient time for medical specialists to start medication in order to avoid seizure. We have also classified the EEG data of patients during ictal state and it has been observed that true positive rate (TPR) is $88.90 \%$.
\end{abstract}

Key words: Epilepsy prediction, pre-ictal state, electroencephalogram (EEG), classification.

\section{Introduction}

Epilepsy [1] is a disease in which patients undergoes seizures caused due to brain functionality disorder. Mostly affected people from this disease belongs to developing countries and children under 15 year of age are in greater percentage. There is no method devised to control seizures, however early diagnosis of epilepsy can help in controlling this problem. Major causes of epilepsy are still unknown. Normally, it is diagnosed in such a way that the patient affected from it is admitted to the hospital and EEG of patient is recorded for many hours sometimes for whole night. These recording are observed and on the basis of these recording doctor diagnoses whether patient is having epilepsy or not. This method of diagnosing epilepsy is not efficient as it requires long wait of admitting and recording EEG of patient. There are several other methods which uses EEG signals to diagnose epilepsy.

Epileptic seizures [2] can cause temporary loss of consciousness, body movements can be violent and repeated jerks that can increase injury risks. One of the most danger aspect of epileptic seizure is that they are not predictable that can limit patient's activities and can disturb their normal routine tasks badly. Surgery and medication are the two common ways of treatments of such patients. However, these treatments provide satisfactory results in around $70 \%$ of the patients whereas $30 \%$ patients could not get control over seizures 
[3]. In many cases surgery is not possible as there is problem locating the cause of epilepsy in a specific area of brain. From this we can conclude that these methods are not as reliable as we need. Therefore, a reliable method of controlling these seizures will be predicting of seizures before they actually happen.

Another important state of epilepsy from EEG is ictal state during which epilepsy takes place. Our task is to predict the pre-ictal state [4] and also detect the ictal state of epilepsy. There are many studies of research that has aimed to determine the pre-ictal time on the basis of EEG. However only few have proposed any reliable methods for determining the pre-ictal state. Ictal state can be detected by classification of seizure [5] data with non-seizure data.

Several features from EEG signals of patients are extracted and then these features are used to train a classifier. Upon testing this classifier gives results whether the patient is affected from epilepsy or not. There can be several classifiers which can be used for classification of EEG signals for epilepsy detection which includes nearest neighbor classifier, k-nearest neighbor classifier, naïve Bayes classifier, support vector machine and artificial neural networks etc.

\section{Existing Methods for Prediction of Epilepsy}

In [6], authors have computed pre-ictal time in EEG datasets for epilepsy. They have computed histogram of different intervals of EEG epochs. Then observations of last 5 minutes are compared with reference data of both pre-ictal and inter-ictal states. Similarity and dissimilarity indexes are measured on the basis of Gaussian Mixture model. A specific threshold is set for every patient after combining this similarity and dissimilarity indexes into a single combined index. This threshold measures the pre-ictal state of the patient affected from seizure. They have computed that epilepsy can be predicted by using this threshold based method with the help of Gaussian mixture model with time of 22.5 minutes before the start of ictal state of seizure. They have also classified EEG data into seizure and normal data with the sensitivity of $88.34 \%$. These results are based on their algorithm applied on dataset of 20 patients with 86 seizures.

In [7], authors have proposed an algorithm from invasive EEG datasets. Their algorithm is known as Advance seizure prediction via preictal re-labelling. This algorithm has predicted seizure 1 to 20 minutes before start of ictal state.

\section{The Dataset}

We have used CHB-MIT [8] datasets of EEG recording of epilepsy patients which is publically available dataset from physionet.org. It is a 23 channels EEG dataset which consists of EEG recordings of 24 patients. The data is available in edf (European data format) format. The data is samples at the rate of $256 \mathrm{~Hz}$. We have considered only those sessions that have seizures.

\section{Univariate Features}

EEG dataset consists of 23 channels and recordings of 24 hours of each patient which is samples at 256 Hz. We have extracted several univariate features from above mentioned dataset that includes maximum value, variance, skewness, inter quartile relation, standard deviation, mean absolute deviation, mutual information, entropy [9], percentile, kurtosis and Hjorth parameter including complexity and mobility. Some features are used for predicting pre-ictal states that include kurtosis, skewness, variance and Hjorth parameters [10] mobility and complexity. Whereas, rest of the features have been used to detect ictal state [11] or classification between seizure and non-seizure EEG data.

Dataset is in edf format; therefore, we have converted it into mat files by using edfread [12] function of MATLAB. As the data is samples at $256 \mathrm{~Hz}$, we have not divided the data into seconds instead we have divided data into frames of 1000 and 2000 data points which are approximately equal to 4 and 8 seconds. 
We have used 1000 frame length in order to detect ictal state whereas 2000 frame length for predicting pre-ictal state.

\section{Proposed Method for Prediction of Epileptic Seizures}

We have proposed an efficient method of predicting epileptic seizures. In our proposed algorithm we have calculated pre-ictal state which is the state before the onset of seizure in EEG data. We have extracted various univariate features including kurtosis, variance, skewness, Hjorth parameters including complexity and mobility. It has been observed that values of kurtosis increases during the pre-ictal state whereas variance decreases during pre-ictal state. Other parameters like skewness, mobility and complexity also increases during this state. We have applied threshold on these features and based on the results of this thresholding we have detected the start of pre-ictal state in the patient's EEG recordings.

It has been observed that by using above mentioned features we are able to predict seizure in EEG data 33.9 minutes prior to start of ictal state of seizure. It means that there is a sufficient time of pre-ictal state for start of medication in order to avoid the seizure.

\section{Classifiers for Detecting Ictal State}

\subsection{Naïve Bayes Classifier}

Any kind of classifier is a function which maps feature vector to class labels as output. Its output is just a label which represents class of that feature vector of test sample. Naïve Bayes classifier [13] assumes that all features are independent conditionally. Normally, features are not independent as they might have dependency but still this classifier provides a best fit classification model. Naïve Bayes classifier uses conditional probability and Bayes theorem assuming that all features are independent. By using Bayes theorem, we can formulate the following:

$$
\text { Posterior }=\frac{\text { Prior } x \text { Likelihood }}{\text { Evidence }}
$$

If $C_{j}$ is the class and $x$ is the feature, then we can also write the posterior in the form of following expression.

$$
P\left(C_{j} \mid x\right)=\frac{P\left(C_{j}\right) P\left(x \mid C_{j}\right)}{P(x)}
$$

Posterior probability of independent features is computed by using above expression which is known as Naïve Bayes classifier. Every time a test sample comes, it is assigned label of that class for which its posterior is greater.

\section{2. $K$ - Nearest Neighbor Classifier}

Nearest neighbor classifier [14] is a classifier which computes distance of test features from training class features and on the basis of shortest distance it assigns label of that class to new test sample. This classifier works well but there is problem if test sample is an outlier then there is a possibility that it is misclassified. In order to solve this problem, $k$-nearest neighbor classifier is designed which computes distance of test sample features with $k$ nearest samples of training class and on the basis of majority votes it assigns class label to test sample. Values of $K$ may be odd values starting from 1 . If value of $k$ is set to 1 then it becomes simple nearest neighbor classifier. It uses simple Euclidean distance for computing distance of 
features from training class. Suppose if $x_{i}$ and $y_{i}$ denotes features of test sample whereas $x_{j}$ and $y_{j}$ denotes features of training class and $D(i, j)$ represents distance between them, then Euclidean distance can be computed using following expression.

$$
D(i, j)=\sqrt{\left(x_{i}-x_{j}\right)^{2}-\left(y_{i}-y_{j}\right)^{2}}
$$

\subsection{Support Vector Machines}

Support vector machines (SVM) [15] is classifier is s supervised learning classifier. It forms higher dimensions planes or hyper planes of features to form a best fit model. SVM can form linear as well as non-linear models. If linear models are used then mapping of features of finite dimensions into higher dimension make these features separable. Originally, it was designed as linear classifier but later it was modified to make it as non-linear classifier as well. SVM can be used as non-linear classifier which forms a model with non-linear boundaries by using kernel. These kernels include, Polynomial, radial basis Gaussian, multilayer perceptron and quadratic. If polynomial kernel is used then by default its order is set to 3 , however its order can be changed. Similarly if radial basis Gaussian (rbf) [16] or multilayer perceptron (mlp) [17] is used as kernel then sigma value of rbf or parameters values of mlp can be changed.

\section{Classification for Ictal State}

We propose also proposed an algorithm for detecting ictal state where seizure actually occurs. In this algorithm, features are extracted from EEG data and labels are assigned to seizure affected EEG data and non-seizure data. Seizure data is assigned label 1 whereas non-seizure data is assigned label 0 . EEG dataset is available in edf format. Edfread function is used to read edf files in matlab. This results in .mat files of EEG data. Data consists of 23 channels and 921000 records. We divide this data into chunks of 1000 . Several features such as mean, minimum point value, standard deviation, entropy, inter quartile range, kurtosis and mean absolute deviation are extracted from publically available dataset on pyshionet.org. Flow diagram of proposed algorithm is shown in following figure.

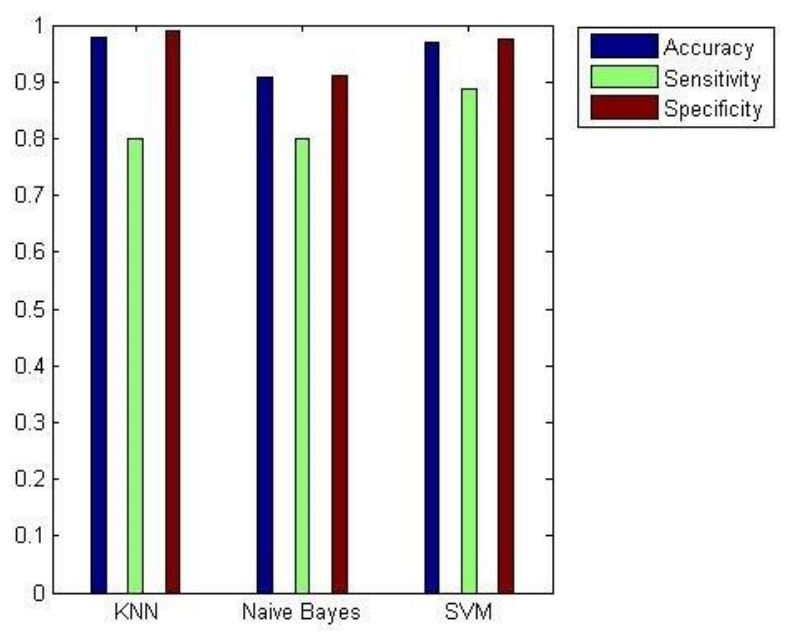

Fig. 1. Comparison of classifiers for detection of ictal state.

Our dataset includes data of 24 patients with seizures in different intervals. EEG of patients is recorded and is stored in edf format. In the first step, edfread function is used in order to read edf files into matlab. Matlab will return mat files which are further used for processing. mat files of EEG recordings include 23 channels. Data is split into chunks of 1000 where each chunk having 23 channels. Seven features are 
extracted from these chunks of data for all 23 channels. This will return $1 \mathrm{x} 161$ feature vector.

Once features are extracted, they are trained by using k-nearest neighbor classifier with different values of $\mathrm{k}$ from 1 to 20 . K-nearest neighbor classifier gives maximum accuracy and true positive rate at $\mathrm{k}=3$. Similarly, features are trained using Naïve Bayes classifier and Support vector machines. For k-nearest neighbor data is shuffled, trained and then tested by using leave one out cross validation. In case of naïve Bayes also leave one out cross validation is used and testing accuracy is computed for every fold. Support vector machine is also trained and tested using leave one out cross validation. Radial basis Gaussian function (rbf) is used as kernel for support vector machine in order to get non-linear decision boundary. Sigma value for rbf is set to 24 . We have performed leave one out cross validation because we have very small data of class 1 objects. Therefore, we cannot apply k-fold cross validation.

Accuracy, sensitivity and specificity for all classifier $k$-nearest neighbor, Naïve Bayes and Support vector machines are computed. Comparison bars are plotted for all three classifiers.

\section{Results}

It has been observed that pre-ictal time for prediction of epileptic seizure is 33.9 minutes which is far better than observed by using existing methods. Five univariate features have been used for prediction of seizure. Our prediction algorithm provides sufficient time for the medication of affected patients in order to avoid the seizure. We have also detected ictal time by classifying the EEG data with the help of three classifiers. We have used seven features that are fed into three different classifiers in order to determine whether seizure occurred or not. It has been observed that support vector machines (SVM) performs better among three classifiers k-nearest neighbor, naïve Bayes and support vector machines. Accuracy of SVM has been $97.07 \%$ with the true positive rate of $88.89 \%$.

Table 1 gives comparison of accuracy and sensitivity among three classifiers. Fig. 1 graphically represents performance measures of three classifiers for the detection of ictal state of Epilepsy in EEG data showing the superior performance of the proposed method.

Table 1. Comparisons of Classifiers for Ictal States

\begin{tabular}{llll}
\hline \hline Classifier & Accuracy & Sensitivity & Specificity \\
\hline$K$ - nearest neighbor & $97.94 \%$ & $80.00 \%$ & $98.86 \%$ \\
Naïve Bayes & $90.66 \%$ & $80.00 \%$ & $91.21 \%$ \\
Support Vector Machines & $97.07 \%$ & $88.89 \%$ & $97.49 \%$ \\
\hline \hline
\end{tabular}

\section{Conclusion}

It has been concluded that by using our proposed algorithm for prediction of epileptic seizure provides sufficient time on average around 33.9 minutes prior to start of ictal state of seizure in EEG data. This pre-ictal time is quite useful in a way that medication can be provided to patient that can avoid seizure and it helps the patient to continue daily tasks and routines. Moreover, it can also avoid loss of consciousness that can reduce the risk of injury involved due to non-consciousness. Ictal state is also detected by classification that can help in surgical treatments of patients.

\section{References}

[1] Acharya, U. R., Sree, S. V., Swapna, G., Martis, R. J., \& Suri, J. S. (2013). Automated EEG analysis of epilepsy: A review. Knowledge-Based Systems, 45, 147-165.

[2] Fisher, R. S., Boas, W. V. E., Blume, W., Elger, C., Genton, P., Lee, P., \& Engel, J. (2005). Epileptic seizures and epilepsy: Definitions proposed by the international league against epilepsy (ILAE) and the international bureau for epilepsy (IBE). Epilepsia, 46(4), 470-472. 
[3] Guenot, M. (2004). Surgical treatment of epilepsy: Outcome of various surgical procedures in adults and children. Revue Neurologique, 160.

[4] Stacey, W., Le Van Quyen, M., Mormann, F., \& Schulze-Bonhage, A. (2011). What is the present-day EEG evidence for a preictal state? Epilepsy Research, 97(3), 243-251.

[5] Bajaj, V., \& Pachori, R. B. (2012). Classification of seizure and nonseizure EEG signals using empirical mode decomposition. IEEE Transactions on Information Technology in Biomedicine, 16(6), 1135-1142.

[6] Zandi, A. (2013). Predicting epileptic seizures in scalp EEG based on a variational bayesian gaussian mixture model of zero-crossing intervals. IEEE Transactions on Biomedical Engineering, 60(5), 1401-1413.

[7] Moghim, N., \& Corne, D. W. (2014). Predicting Epileptic Seizures in Advance.

[8] Moody, G., Roger, G. M., \& Ary, L. G. (2011). PhysioNet: Physiologic signals, time series and related open source software for basic, clinical, and applied research. Proceedings of Annual International Conference of the IEEE Engineering in Medicine and Biology Society.

[9] Acharya, U. R. (2012). Automated diagnosis of epileptic EEG using entropies. Biomedical Signal Processing and Control, 7(4), 401-408.

[10] Mormann, F., Kreuz, T., Rieke, C., Andrzejak, R. G., Kraskov, A., David, P., Elger C. E., \& Lehnertz, K. (2005). On the predictability of epileptic seizures. Clinical Neurophysiology, 116, 569-587.

[11] Osorio, I., Mark, G. F., \& Steven, B. W. (1998). Real-time automated detection and quantitative analysis of seizures and short-term prediction of clinical onset. Epilepsia, 39(6), 615-627.

[12] De Miras, J. R. (2016). Fractal analysis in MATLAB: A tutorial for neuroscientists. The Fractal Geometry of the Brain, 523-532.

[13] Jordan, A. (2002). On discriminative vs. generative classifiers: A comparison of logistic regression and naive bayes. Advances in Neural Information Processing Systems, 14, 841.

[14] Cunningham, P., \& Sarah, J. D. (2007). K-Nearest neighbour classifiers. Multiple Classifier Systems, 1-17.

[15] Hearst, M. (1998). Support vector machines. IEEE Intelligent Systems and Their Applications, 13(4), 18-28.

[16] Chen, S., Cowan, C. F., \& Grant, P. M. (1991). Orthogonal least squares learning algorithm for radial basis function networks. IEEE Transactions on Neural Networks, 2(2), 302-309.

[17] Gardner, M. W., \& Dorling, S. R. (1998). Artificial neural networks (the multilayer perceptron) - A review of applications in the atmospheric sciences. Atmospheric Environment, 32(14), 2627-2636.

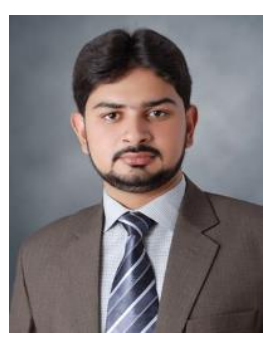

Syed Muhammad Usman was born in 1989 in Punjab, Pakistan. He received the B.S in computer system engineering from Islamia University of Bahawalpur, Pakistan in 2010. Currently, he is doing M.S in computer engineering from College of Electrical and Mechanical Engineering, NUST, Islamabad. He is also teaching different courses of computer science and IT in NUML, Islamabad. His research interests include machine learning, biomedical signal processing, pattern recognition and analysis of EEG signals for epilepsy diagnosis.

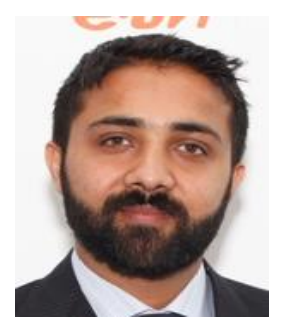

Ali Hassan received his B.E. and M.S. degrees in computer engineering from the National University of Sciences and Technology, College of Electrical and Mechanical Engineering, Pakistan, in 2004 and 2007. He received the Ph.D. degree in electrical engineering from the University of Southampton, UK, in 2012. He is currently working at NUST College of Electrical and Mechanical Engineering as an assistant professor at the Department of Computer Engineering. His research interests include application of machine learning to speech and image processing in the domains of speech, texture classification and biomedical engineering. 